\title{
Effects of Intratympanic Injection of Isosorbide on the Vestibular Function of Animal Models of Endolymphatic Hydrops
}

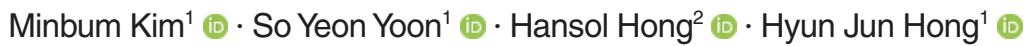 \\ ${ }^{1}$ Department of Otorhinolaryngology-Head and Neck Surgery, Catholic Kwandong University College of Medicine, Incheon; \\ ${ }^{2}$ Department of Otorhinolaryngology, Yonsei University College of Medicine, Seoul, Korea
}

Objectives. The aims of this study were to investigate the effects of intratympanic injections of isosorbide on vestibular function in animal models of endolymphatic hydrops and to find a new treatment option for the acute onset of vertigo in Ménière disease (MD).

Methods. Seventy male guinea pigs received intratympanic injection of isosorbide (IT-ISB). The animals were divided into three study groups: control, a chronic hydrops model, and an acute hydrops model. Intracochlear drug concentrations were measured using high-performance liquid chromatography. Vestibular function was analyzed using an animal rotator test with bidirectional sinusoidal harmonic acceleration before and after IT-ISB administration. Histological changes were also investigated.

Results. ISB successfully permeated the perilymph through the round window membrane (RWM) at all three concentrations $(25 \%, 50 \%$, and $100 \%)$. In the chronic hydrops model, while IT-ISB histologically induced a reduction of endolymphatic hydrops, vestibular function was unchanged. In the acute hydrops model, no endolymphatic hydrops was histologically observed, and vestibular symmetry was also preserved after IT-ISB.

Conclusion. ISB passed through the RWM into the perilymphatic space even at lower concentrations. IT-ISB histologically reduced hydrops in the chronic model and preserved symmetrical vestibular function in the acute model. IT-ISB could be a treatment candidate for acute attacks of vertigo in MD.

Keywords. Isosorbide; Endolymphatic Hydrops; Meniere Disease

\section{INTRODUCTION}

The treatment of Ménière disease (MD) has progressed slowly, although it has been the focus of many studies, and no treatment of choice has yet been established to inhibit the progression of the disease [1]. As its pathophysiology has yet to be elu-

\footnotetext{
- Received December 16, 2019

Revised August 31, 2020

Accepted October 1, 2020

- Corresponding author: Hyun Jun Hong

Department of Otorhinolaryngology-Head and Neck Surgery, Catholic Kwandong University College of Medicine, 25 Simgok-ro 100beon-gil, Seo-gu, Incheon 22711, Korea

Tel: +82-32-290-3939, Fax +82-32-290-3050

E-mail: hyunjun.hong@gmail.com
}

cidated, the aim of management is still not curative; instead, symptomatic treatment aims to reduce the occurrence of vertigo and the risk of hearing impairment. Moreover, treatment algorithms for MD vary from one center to another even in the same country [2].

Osmotic diuretics have recently been used for the treatment of MD on the basis of studies indicating that these agents can reduce endolymphatic hydrops, based on histological, biochemical, and electrophysiological evidence of efficacy [3-7]. In particular, isosorbide (ISB) reduced cochlear endolymph volume without a prominent rebound phenomenon, unlike glycerol, in several studies [7-9]. Its clinical efficacy has been reported for the relief of symptoms of dizziness, headache, and tinnitus [3]. Hearing improvement was also observed in some cases after

Copyright @ 2021 by Korean Society of Otorhinolaryngology-Head and Neck Surgery.

This is an open-access article distributed under the terms of the Creative Commons Attribution Non-Commercial License (https://creativecommons.org/licenses/by-nc/4.0)

which permits unrestricted non-commercial use, distribution, and reproduction in any medium, provided the original work is properly cited. 


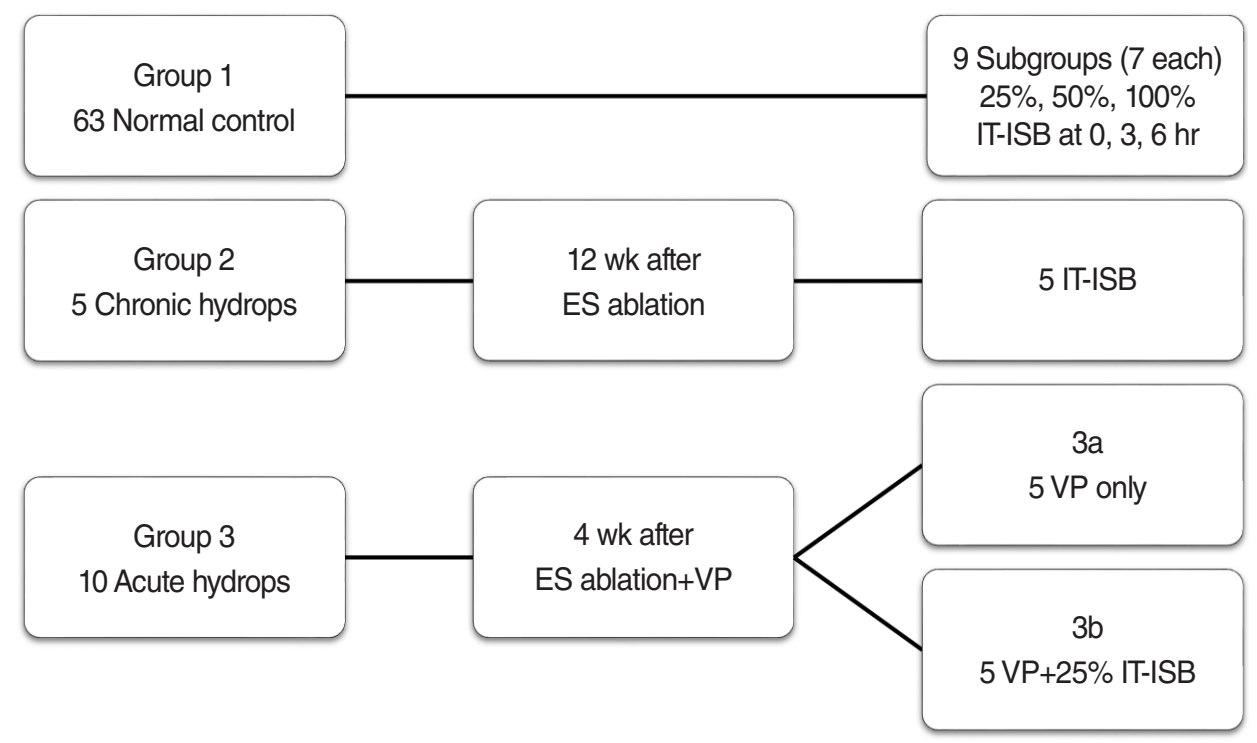

Fig. 1. Experimental groups. Group 1, the control group, was injected with three different isosorbide concentrations (IT-ISB). Group 2, the chronic hydrops model, was treated with IT-ISB at 12-16 weeks after endolymphatic sac (ES) ablation surgery. Group 3, the acute hydrops model, was treated with (3a) desmopressin (VP) injected at 2-4 weeks after ES surgery for the induction of hydrops aggravation and (3b) ITISB along with VP. IT-ISB, intratympanic injection of isosorbide.

oral administration $[5,10]$. For these reasons, it has been used as a therapeutic agent for MD in Japan and Korea [3,11-13].

Although the oral administration of ISB (PO-ISB) improved endolymphatic hydrops in an animal model [7] and showed clinical efficacy in patients, it has several systemic adverse effects, including nausea and headache $[12,14]$. Therefore, local application of ISB is considered promising for the treatment of $\mathrm{MD}$, as it can be delivered at higher doses without systemic side effects. In our previous study, ISB successfully passed through the round window membrane (RWM) after intratympanic injection (IT-ISB) and showed higher intracochlear concentrations in the perilymph than after oral administration [13]. However, no study has examined the efficacy of IT-ISB in an animal model.

To study the effect of IT-ISB on endolymphatic hydrops, it is critical to use an appropriate animal hydrops model. Although the traditional chronic model using endolymphatic sac surgery $[7,15]$ has been well studied, it can only simulate the final stage

\section{H I G H L I G H T S}

- No treatment of choice has been established for acute attacks of vertigo in Ménière disease.

- Isosorbide passed through the round window membrane even at lower concentrations.

- Intratympanic injection of isosorbide histologically reduced hydrops in the chronic model and preserved symmetrical vestibular function in the acute model.

- Intratympanic injection of isosorbide could be a treatment option for acute attacks of vertigo in Ménière disease. of MD, with irreversible hearing loss and vestibular dysfunction. Recently, a new acute model of the acute aggravation of hydrops, induced by vasopressin, was introduced [16], and its vestibular function was investigated [17]. In this study, for the first time, we investigated whether IT-ISB can reduce endolymphatic hydrops and preserve symmetric vestibular function using both the traditional chronic model and the dynamic acute model.

\section{MATERIALS AND METHODS}

\section{Animals and experimental groups}

A total of 78 male guinea pigs with Preyer's reflex (Hartley guinea pigs, $350-400 \mathrm{~g}$ ) were used. We divided the animals into three study groups as follows (Fig. 1): group 1, the control group, consisting of healthy animals treated with three different IT-ISB concentrations $(n=63)$; group 2 , the chronic hydrops model, consisting of animals treated with IT-ISB at 12-16 weeks after endolymphatic sac ablation surgery $(\mathrm{n}=5)$; and group 3 , the acute hydrops model, consisting of animals treated with (1) desmopres$\sin \left(\mathrm{VP}\right.$; [deamino-Cys ${ }^{1}, \mathrm{D}-\mathrm{Arg}^{8}{ }^{8}$-vasopressin acetate salt hydrate; Sigma-Aldrich, St. Louis, MO, USA) injected at 2-4 weeks after surgery for the induction of hydrops aggravation $(n=5)$ and (2) IT-ISB along with VP $(n=5)$.

The group 1 was divided into nine subgroups according to collection time of perilymph (0, 3 , and 6 hours) and drug concentration $(25 \%, 50 \%$, and $100 \%)$ as below. Considering the loss due to the invasive perilymph sampling, seven animals were assigned to each subgroups. For anesthesia induction, xylazine $(5 \mathrm{mg} / \mathrm{kg}, \mathrm{IM})$ and ketamine $(35 \mathrm{mg} / \mathrm{kg}$, IM) were administered. 
This study was approved by the University Animal Care and Use Committee (IACUC 150617-365).

\section{Surgical ablation of the endolymphatic sac and induction of acute aggravation of endolymphatic hydrops}

As described in the previous study [17], the endolymphatic sac was partially cauterized with a bipolar electrocoagulator via the suboccipital approach. For the chronic hydrops model, we waited for 12-16 weeks after surgery and injected $100 \mu \mathrm{g} / \mathrm{kg}$ of VP subcutaneously at 2-4 weeks after surgery for the acute hydrops model of an early stage in accordance with the method described in a previous study [17].

\section{Administration of ISB}

For IT-ISB, the bulla was opened with cutting burrs to expose the round window via the retro-auricular approach as in a previous study [13]. ISB solutions of $0.2-0.3 \mathrm{~mL}$ of $25 \%, 50 \%$, and $100 \%$ ISB (Kowa Co., Nagoya, Japan) was applied onto the RWM with a 1-mL syringe fitted with a 30-G spinal needle, filling the whole middle ear cavity. After 30 minutes, the middle ear was rinsed with $10 \mathrm{~mL}$ of phosphate-buffered saline. For the normal control group, IT-ISB of different drug concentrations was also administered (25\%, 50\%, and $100 \%$ IT-ISB).

Unexpected spontaneous nystagmus, beating to the non-perfusion ear side, was observed (Supplementary Material 1, Video Clip 1; https://youtu.be/Kp99mZTTKNs) after 50\% and 100\% IT-ISB. To prevent spontaneous nystagmus due to the ototoxicity of ISB, which can interfere with the exact analysis of vestibular function, 25\% IT-ISB was used in the study for the animal model groups (group 2,3).

\section{Perilymph collection through the RWM (group 1)}

After incision of the RWM, 2- $\mu \mathrm{L}$ perilymph was gently harvested with an ultra-fine pipette tip (Multimax 0.1-10 $\mu \mathrm{L}$ long-reach tip; Sorenson Bioscience, Salt Lake City, UT, USA) attached to a micropipette to avoid damage to the basilar membrane [13]. According to collection time of perilymph $(0,3$, and 6 hours) and drug concentration $(25 \%, 50 \%$, and $100 \%)$, perilymph was sampled in each nine subgroup of seven animals. Eight animals were died during the surgical procedures, and 11 perilymph samples was excluded because of blood clotting and contamination at the stage of preparation. Thus, we finally analyzed intracochlear concentration of 44 samples.

\section{Measurement of intracochlear concentration according to ISB concentration}

For chromatographic separation of ISB and analysis of its intracochlear concentrations, high-performance liquid chromatography coupled with refractive index detection (HPLC-RI) was used. Each sample was diluted with $28 \mu \mathrm{L}$ of distilled water and filtered through a regenerated cellulose filter $(0.2-\mu \mathrm{m}$; Sartorius Co., Goettingen, Germany). The Acme 9000 HPLC system was equipped with RI750F RID (YL Instrument Co., Anyang, Korea) and an Aminex HPX-87H Column (Bio-Rad, Hercules, CA, USA). A mobile phase of $0.005 \mathrm{M}$ sulfuric acid and a flow rate of $0.6 \mathrm{~mL} / \mathrm{min}$ were applied. Data were acquired and analyzed with the Autochro-3000 chromatography processing program (YL Instrument Co.). To avoid error from blood contamination during sampling, the glucose concentration was simultaneously analyzed. As the previously reported normal glucose level in the perilymph [18] was $3.5 \pm 1.0 \mathrm{mM}$, any samples with a glucose level of $>5.5 \mathrm{mM}$ were regarded as contaminated with plasma, with a glucose level of $7.3 \pm 0.9 \mathrm{mM}$. After IT-ISB with $25 \%, 50 \%$, and $100 \%$ concentrations, intracochlear concentrations were compared. The Kruskal-Wallis test and Mann-Whitney $U$-test with Bonferroni correction were performed to determine statistical differences. A Bonferroni corrected $P$-value of $<0.05$ was considered significant.

\section{Vestibular function test}

To evaluate vestibular function, sinusoidal harmonic acceleration (SHA) with a bidirectional $60^{\circ}$ rotation at a frequency of $0.2 \mathrm{~Hz}$ was conducted using a customized animal rotator after attaching a fluorescent marker on each pupil under topical anesthesia as in a previously reported study [11]. Nystagmus was recorded using a charge-coupled device camera, and video analysis of bidirectional eye velocity was performed. Data were collected from at least five cycles, and the maximum slow-phase velocity (SPV) of the horizontal eye movement was averaged. Bidirectional symmetry score (\%) was defined as the ratio of maximum SPV with right- and left-directional rotations. Vestibular function was measured at baseline and 1 hour after IT-ISB in all three groups. The Wilcoxon signed-rank test was performed to determine statistical differences between the symmetry scores measured before and after IT-ISB, and a $P$-value of $<0.05$ was considered significant.

\section{Histological observation of endolymphatic hydrops}

After fixation in 4\% formaldehyde solution for 2 days, decalcification with ethylenediamine tetra-acetic acid was performed for approximately 4 weeks, followed by dehydration and embedding in paraffin. Blocks were sectioned parallel to the modiolar axis at a 6-mm thickness and stained with hematoxylin and eosin. Changes of Reissner's membrane and endolymphatic space were observed under light microscopy.

\section{RESULTS}

\section{IT-ISB in the normal controls (group 1) Intracochlear ISB concentrations after injection at different ISB concentrations}

ISB successfully permeated into the perilymph through the RWM after injection at all three concentrations $(25 \%, 50 \%$, and $100 \%)$. 
The concentrations in the perilymph were $59.77 \pm 25.79 \mathrm{mM}$ immediately $(n=5), 19.28 \pm 4.02 \mathrm{mM}$ at 3 hours $(n=7)$, and $13.56 \pm$ $4.38 \mathrm{mM}$ at 6 hours $(\mathrm{n}=4)$ after $25 \%$ IT-ISB; $213.13 \pm 106.65$ $(\mathrm{n}=4), 152.42 \pm 24.33(\mathrm{n}=5)$, and $40.72 \pm 22.87 \mathrm{mM}(\mathrm{n}=7)$, respectively, after 50\% IT-ISB; and 245.47 $\pm 112.84(\mathrm{n}=4), 149.80 \pm$ $65.39(\mathrm{n}=4)$, and $66.81 \pm 20.41 \mathrm{mM}(\mathrm{n}=4)$, respectively, after $100 \%$ IT-ISB. The intracochlear concentration of $50 \%$ IT-ISB was higher than that of $25 \%$ IT-ISB at 0,3 , and 6 hours $(P=0.03$, $P<0.01$, and $P<0.01$, respectively). The intracochlear concentration of $100 \%$ IT-ISB was higher than that of $25 \%$ IT-ISB at 0,3 ,

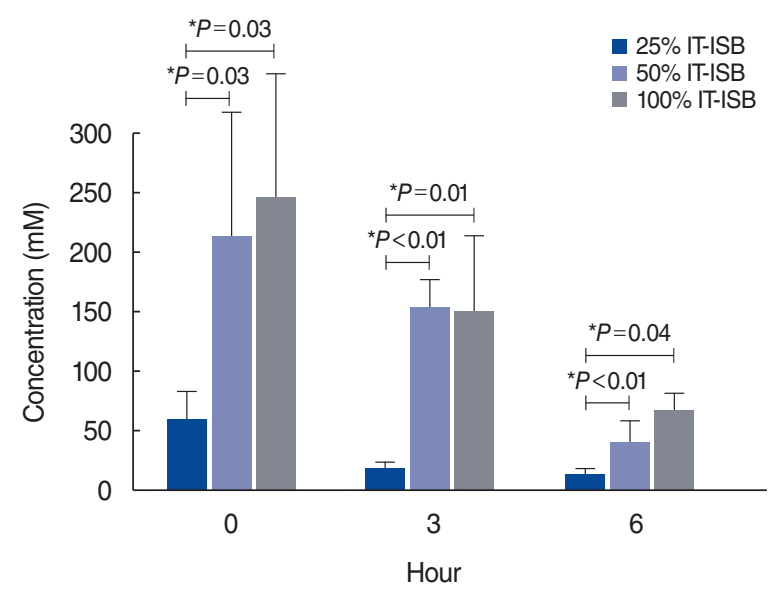

Fig. 2. Intracochlear concentrations of isosorbide after injections at different concentrations. Whiskers show the standard deviations. ITISB, intratympanic injection of isosorbide. ${ }^{*} P<0.05$.

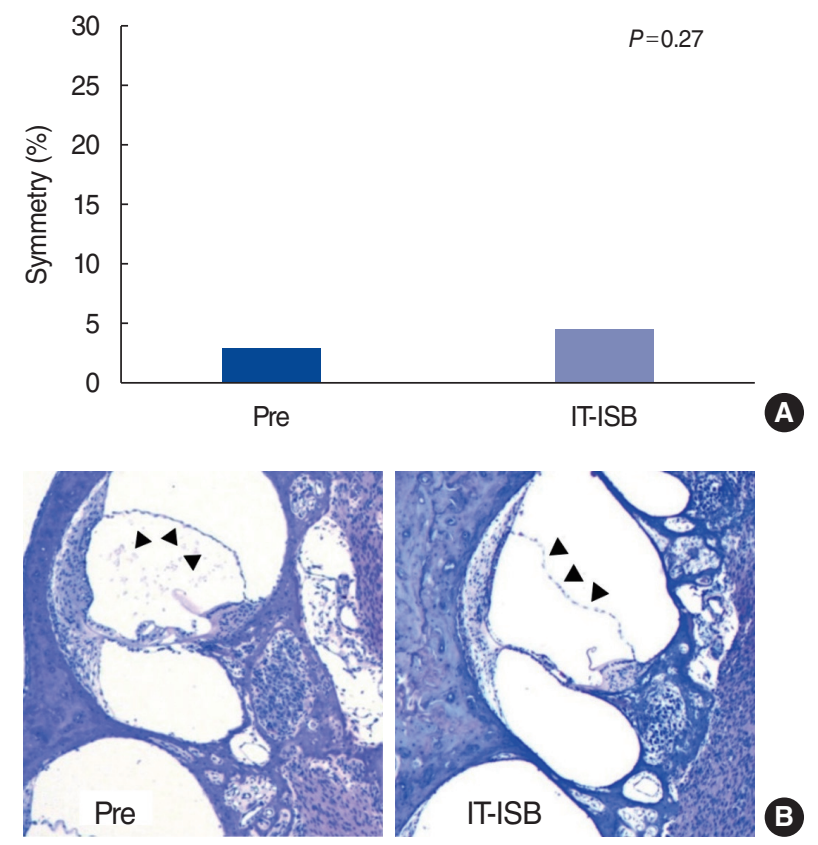

Fig. 3. Intratympanic injection of isosorbide (IT-ISB) in the chronic hydrops model. (A) No significant change of symmetrical vestibular function was observed after IT-ISB. (B) Endolymphatic hydrops decreased after IT-ISB (arrowheads) (H\&E, ×40). and 6 hours $(P=0.03, P=0.01$, and $P=0.04$, respectively). No significant differences were observed between $50 \%$ and $100 \%$ IT-ISB at 0,3 , and 6 hours $(P>0.05, P>0.05$, and $P>0.05$, respectively) (Fig. 2).

\section{Vestibular function change after different ISB concentrations}

After 50\% IT-ISB, unexpected spontaneous nystagmus was observed for 6 to 9 hours in all animals. By contrast, no spontaneous nystagmus was observed after $25 \%$ IT-ISB, and the symmetry score $(4.96 \% \pm 4.68 \%)$ was within the normal range (range,
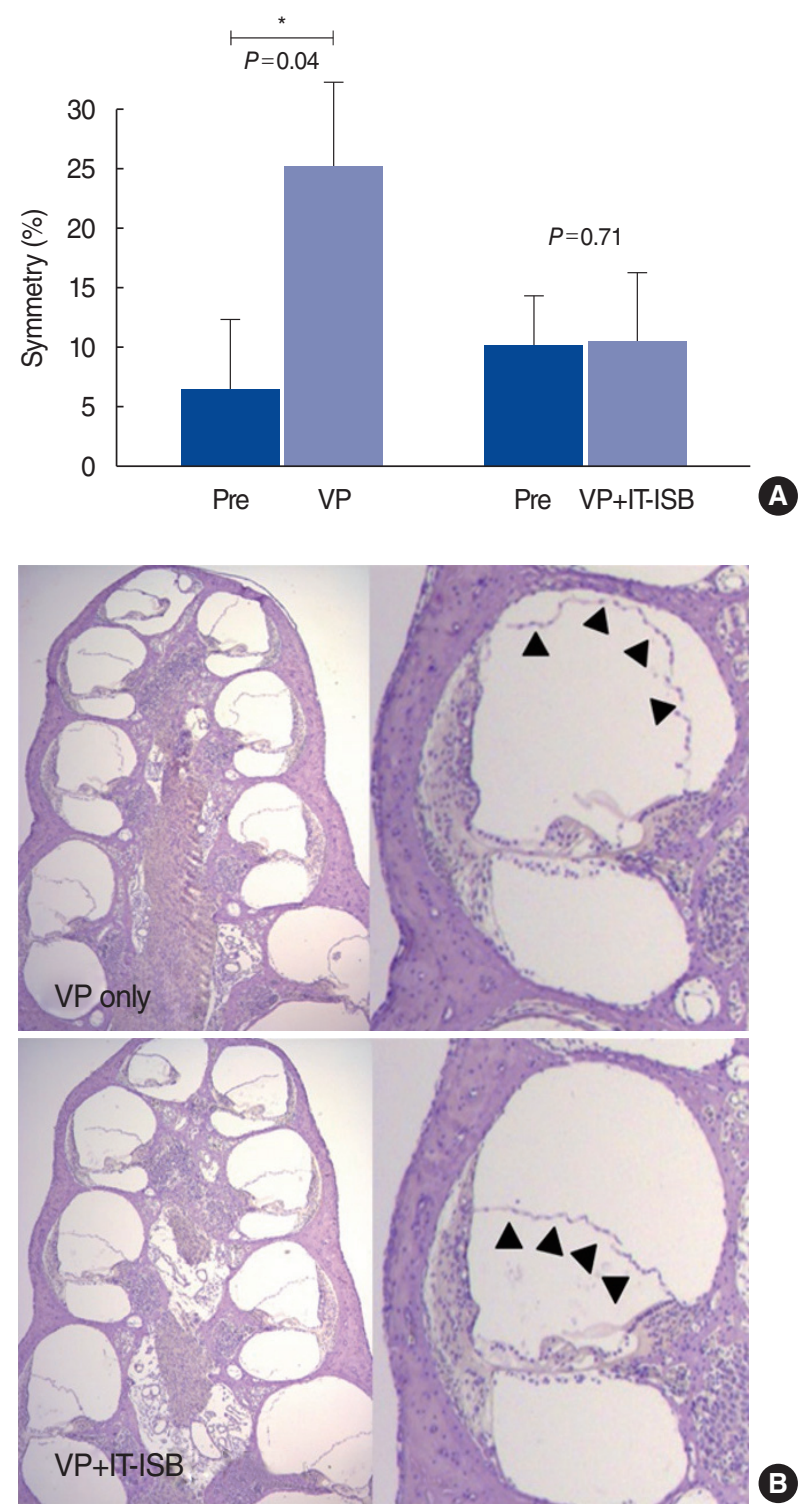

Fig. 4. Intratympanic injection of isosorbide (IT-ISB) in the acute hydrops model. (A) Acute vestibular dysfunction after the induction with desmopressin (VP) and preserved vestibular symmetry after ITISB along with VP. Whiskers show the standard deviations. ${ }^{*} P<0.05$. (B) Acute endolymphatic hydrops after VP, however, preserved normal endolymphatic space after IT-ISB along VP (arrowheads) (H\&E, $\times 40)$. 
$0.47 \%-11.22 \%)$

\section{IT-ISB in the chronic hydrops model (group 2)}

At 16 weeks after ES ablation, symmetrical response was observed in the bidirectional SHA tests $(2.94 \% \pm 1.66 \%$; range, $1.26 \%$ $4.89 \%$ ) (Fig. 3A). Extension of the scala media with stretching of Reissner's membrane was observed in two of the five animals on histological examination (Fig. 3B). After 25\% IT-ISB, the symmetrical response was unchanged $(4.62 \% \pm 2.65 \%, P=0.27)$ (Fig. 3A). By contrast, Reissner's membrane appeared convex in a histological section (Fig. 3B).

\section{IT-ISB in the acute hydrops model (group 3) Confirmation of VP-induced aggravation of hydrops (group 3a)}

Before VP injection, a symmetrical response was observed in the bidirectional SHA tests (symmetry score, $6.50 \% \pm 7.60 \%$ ). However, approximately 1 hour after the VP injection, the symmetry score increased to $25.23 \% \pm 9.84 \%$ in the bidirectional SHA tests $(P=0.04)$ (Fig. 4A). On histological examination, extension of the scala media with stretching of Reissner's membrane was observed 1 hour afterVP injection in all animals (Fig. 4B).

\section{Effect of IT-ISB on VP-induced aggravation of hydrops (group 3b)}

After $25 \%$ IT-ISB along with VP, the vestibular symmetry was unchanged $(10.18 \% \pm 4.26 \%$ vs. $10.51 \% \pm 8.01 \%, P=0.71)$ (Fig. 4A). In addition, no endolymphatic hydrops was histologically observed, and Reissner's membrane was relaxed and crooked (Fig. 4B).

\section{DISCUSSION}

Experimental [3,5,7] and clinical evidence [12,14] has shown that PO-ISB is an effective drug for MD. In our previous study, we detected ISB and measured its concentration in the perilymph after PO-ISB for the first time [13]. Although reduction of endolymph was confirmed histologically in a guinea pig model, the peak reduction in cochlear endolymph volume occurred 6 hours after oral intake [18]. As vertigo episodes typically last for $20 \mathrm{~min}$ utes to 12 hours in MD [19], PO-ISB might not be an appropriate medication for acute attacks of vertigo. In addition, PO-ISB has systemic adverse effects such as nausea, vomiting, and headache $[12,14]$. Furthermore, ISB may increase antidiuretic hormone levels and plasma osmolality, which may worsen endolymphatic hydrops over the long term [14]. As a result, a high oral dose of ISB is difficult to recommend.

Intratympanic drug injections, including dexamethasone and gentamicin therapy, have been widely used for the treatment of MD [20]. We previously reported that IT-ISB can pass through the RWM and diffuse into the perilymph [13]. In our results, intracochlear ISB was detected immediately after IT-ISB, and a high intracochlear concentration was measured even when the most dilute concentration (25\%) was used. These results suggest that IT-ISB may be a faster and more effective treatment option than PO-ISB for the acute onset of vertigo. Moreover, IT-ISB can reduce systemic adverse effects and allow the use of a higher dose. However, to our knowledge, the efficacy of IT-ISB for endolymphatic hydrops in animal models has not been reported yet.

To study the effects of IT-ISB, we first used the traditional animal model created by surgical ablation of the endolymphatic sac (group 2). Histological decompression of endolymphatic hydrops was observed after IT-ISB. By contrast, we could not find a significant change in vestibular function. We assume that this finding resulted from a pre-existing deterioration of vestibular function and subsequent vestibular compensation in this model. As this model only represents the chronic stage of hydrops, the socalled burnout stage [18], we could not prove whether it was effective for the acute onset of vertigo. However, the main treatment target of MD is not persistent disequilibrium in the chronic stage, but vertiginous episodes in the acute progressive stage. Thus, IT-ISB must be applied in an animal model that can simulate the acute onset of hydrops, as in the progressive mid-stage of MD.

Next, to investigate the effect of IT-ISB on the acute aggravation of hydrops, we used the new vasopressin-induced hydrops model (group 3). In this model, a vasopressin injection is used to induce hydrops aggravation by water shift from the perilymph to the endolymph after surgical ablation of the endolymphatic sac [16], which results in transient vestibular dysfunction [17]. As it only induces vestibular dysfunction for several hours, changes in vestibular function after treatment in the acute stage of hydrops could be evaluated. In our study, IT-ISB preserved both the symmetrical vestibular response and a normal shape of Reissner's membrane even after the vasopressin injection. We assume that IT-ISB can induce a water shift from the endolymph to the perilymph by osmotic pressure [7], which reversed the water shift induced by vasopressin and relieved the aggravation of endolymphatic hydrops. Therefore, IT-ISB is expected to be a treatment candidate for vertigo onset in MD.

There are several limitations of this study. First, the distribution of the animal numbers was substantially unbalanced. Since no previous study investigated changes in intracochlear concentrations over time after IT-ISB, we first had to determine the optimal concentration and test for possible ototoxicity in normal animals before starting IT-ISB in the animal model groups. For this reason, we had to use more animals in the normal animal group than in the animal model groups. Based on the results, it was possible to assign five animals equally to each animal model. Second, we did not investigate the effect of IT-ISB on audiologic function. Since we used the trans-bullar approach and highviscosity ISB for the perfusion, which can substantially affect hearing thresholds, it would have been difficult to measure hearing adequately. To evaluate hearing changes in animal models, it is necessary to conduct a further study using a minimally invasive technique and ISB at a lower viscosity. Third, the present hydrops 
model is not yet perfect for the investigation of MD. Furthermore, the reason for discrepancies between histologic hydrops and MD remains controversial. Individual differences in susceptibility and resistance to MD are also not well understood. Further research is needed to clarify the pathophysiology of MD. In addition, the statistical reliability of our findings might have been low due to a small sample size. A follow-up study is necessary with a larger sample size, as well as an a posteriori power analysis.

Even though we found that IT-ISB could be feasible for the treatment of MD, further research is needed to clarify certain points. First, high ISB concentrations can induce transient vestibular dysfunction. An intratympanic injection of $100 \%$ ISB was reported to provoke paralytic nystagmus in rabbits and cats, even though it diminished in 3 hours [21]. We also observed that 50\% IT-ISB induced spontaneous nystagmus, which suggests the possibility of short-term vestibular toxicity. We need to determine the optimum concentration to avoid unexpected vestibular dysfunction. Second, the safety of IT-ISB for the middle ear mucosa or inner ear structure should be assessed through functional and histological studies before clinical use because ISB has a high viscosity and specific gravity, as well as a dehydrating effect.

In conclusion, ISB passed through the RWM into the perilymphatic space even at lower concentrations. IT-ISB histologically reduced hydrops in the chronic model and preserved symmetrical vestibular function in the acute model. IT-ISB could be a treatment candidate for vertigo in MD.

\section{CONFLICT OF INTEREST}

No potential conflict of interest relevant to this article was reported.

\section{ACKNOWLEDGMENTS}

This research was supported by the Basic Science Research Program through the National Research Foundation of Korea (NRF) funded by the Ministry of Science, ICT and Future Planning (NRF2017R1D1A3B03030968). We wish to express our most sincere gratitude and appreciation to Prof. Akinobu Kakigi for his guidance and advice.

\section{ORCID}

Minbum Kim https://orcid.org/0000-0002-8051-1403

So Yeon Yoon https://orcid.org/0000-0002-7720-6424

Hansol Hong https://orcid.org/0000-0003-2137-2404

Hyun Jun Hong https://orcid.org/0000-0002-7808-7877

\section{AUTHOR CONTRIBUTIONS}

Conceptualization: MK. Data curation: SYY. Formal analysis: MK. Funding acquisition: MK. Methodology: MK, HH, SYY. Project administration: HJH. Visualization: HH. Writing-original draft: MK.Writing-review \& editing: $\mathrm{MK}, \mathrm{HH}, \mathrm{HJH}$.

\section{SUPPLEMENTARY MATERIALS}

Supplementary materials can be found via https://doi.org/10. 21053/ceo.2019.02040.

\section{REFERENCES}

1. Foster CA. Optimal management of Meniere's disease. Ther Clin Risk Manag. 2015 Feb;11:301-7.

2. Nevoux J, Barbara M, Dornhoffer J, GibsonW, KitaharaT, Darrouzet V. International consensus (ICON) on treatment of Meniere's disease. Eur Ann Otorhinolaryngol Head Neck Dis. 2018 Feb;135(1S):S29-32.

3. Kitahara M, Takeda T, Yazawa Y, Matsubara H, Kitano H. Treatment of Meniere's disease with isosorbide. ORL J Otorhinolaryngol Relat Spec. 1982;44(4):232-8.

4. Juhn SK, Prado S, Pearce J. Osmolality changes in perilymph after systemic administration of glycerin. Arch Otolaryngol. 1976 Nov; 102(11):683-5.

5. Kakigi A, TakedaT, Saito H, Kataoka H. Effect of isosorbide on hearing loss due to endolymphatic hydrops. Acta Otolaryngol Suppl. 1995; 519:223-6.

6. TakedaT,Takeuchi S, Saito H. Effect of glycerol on pressure difference between perilymph and endolymph. Acta Otolaryngol. 1990 Jul-Aug; 110(1-2):68-72.

7. Kakigi A, Takeda S, Takeda T, Sawada S, Azuma H, Higashiyama K, et al.Time course of dehydrating effects of isosorbide on experimentally induced endolymphatic hydrops in guinea pigs. ORL J Otorhinolaryngol Relat Spec. 2004;66(6):291-6.

8. Padoan S. Oral versus i.v. administration of the glycerol test: side-effects and usefulness. Acta Otolaryngol. 2003 May;123(4):482-7.

9. Matsubara H, Kitahara M,TakedaT, Yazawa Y. Rebound phenomenon in glycerol test. Acta Otolaryngol Suppl. 1984;419:115-22.

10. Larsen HC,Angelborg C, Klockoff I, Stahle J.The effect of isosorbide and urea on hearing in patients with Meniere's disease. Acta Otolaryngol (Stockh). 1984;412:113-4.

11. Yamazaki T, Imoto T, Hayashi N, Watanabe S, Kozaki H, Abe T. Meniere's disease and isosorbide as an oral hyperosmotic agent. Arch Otorhinolaryngol. 1982;234(1):97-104.

12. Nozawa I, Nakayama H, Hashimoto K, Imamura S, Hisamatu K, Murakami Y. Efficacy of long-term administration of isosorbide for Meniere's disease. ORL J Otorhinolaryngol Relat Spec. 1995 MayJun;57(3):135-40.

13. Kim M, Do KH, Kim KS. Isosorbide concentration in perilymph of the guinea pig after oral administration versus that after round window perfusion. Clin Exp Otorhinolaryngol. 2014 Dec;7(4):281-5.

14. Kakigi A, Takeda T, Sawada S, Taguchi D. Antidiuretic hormone and osmolality in isosorbide therapy and glycerol test. ORL J Otorhinolaryngol Relat Spec. 2006 Aug;68(5):279-82.

15. Klockhoff I. Diagnosis of Meniere's disease. Arch Otorhinolaryngol. 1976 Sep;212(4):309-14.

16. Egami N, Kakigi A, Sakamoto T, Takeda T, Hyodo M, Yamasoba T. 
Morphological and functional changes in a new animal model of Meniere's disease. Lab Invest. 2013 Sep;93(9):1001-11.

17. Kim M, Kim KS. Vestibular function change in a vasopressin-induced hydrops model. Otol Neurotol. 2017 Dec;38(10):e495-500.

18. Salt AN, Plontke SK. Endolymphatic hydrops: pathophysiology and experimental models. Otolaryngol Clin North Am. 2010 Oct;43(5): 971-83.

19. Lopez-Escamez JA, Carey J, Chung WH, Goebel JA, Magnusson M,
Mandala M, et al. Diagnostic criteria for Meniere's disease. J Vestib Res. 2015;25(1):1-7.

20. Sajjadi H, Paparella MM. Meniere's disease. Lancet. 2008 Aug;372 (9636):406-14.

21. Shigeno K, Egami T, Sasano T. Experimental study of nystagmus induced by injecting various solutions into the middle ear cavity. Acta Otolaryngol. 1989 Jul-Aug;108(1-2):31-7. 